\title{
Processing local transitions versus long-distance syntactic hierarchies
}

\author{
Angela D. Friederici \\ Max Planck Institute for Human Cognitive and Brain Sciences, Department of Neuropsychology, PO Box 500 355, 04303 Leipzig, \\ Germany
}

\begin{abstract}
A recent study by Fitch and Hauser reported that finitestate grammars can be learned by non-human primates, whereas phrase-structure grammars cannot. Humans, by contrast, learn both grammars easily. This species difference is taken as the critical juncture in the evolution of the human language faculty. Given the farreaching relevance of this conclusion, the question arises as to whether the distinction between these two types of grammars finds its reflection in different neural systems within the human brain.
\end{abstract}

For centuries people have sought the critical parameters surrounding the evolution of language. With the advent of genetic research methods, the possible role of a part gene, $F O X P 2$, in speech and language abilities has been brought to light [1]. Moreover, it has been shown that this gene was the target of selection during recent human evolution [2]. In a recent paper, Fitch and Hauser [3] suggest that, behaviorally, the crucial juncture in the evolution of the human faculty was the ability to process hierarchical structures. Using a familiarization-discrimination paradigm, they demonstrated that monkeys can learn finitestate grammars (FSG), which can be fully specified by transition probabilities between elements in a sequence, but that they cannot learn phrase-structure grammars (PSG), characterized by their admission of complex hierarchical structures and long-distance dependencies. The stimuli they used in the two types of grammars were sequences of consonant-vowel nonsense syllables (e.g. pa, $l i, m o$ ). These were matched in length, composition, volume and other acoustic features. Each grammar consisted of the same number of syllables of two classes, A and B, but they differed in structure (see Figure 1).

After a familiarization phase of $20 \mathrm{~min}$ with 60 grammar-consistent sequences, monkeys were tested with grammatical and ungrammatical items. They demonstrated clear discrimination between grammatical and ungrammatical sequences for the FSG, but no such discrimination was observed for the PSG. By contrast, humans showed learning for both grammars (with a familiarization phase of under $3 \mathrm{~min}$ ), easily discriminating grammatical from ungrammatical sequences in both the FSG and the PSG.

What constitutes the human language faculty? If the observed difference between the mastery of PSG in humans and monkeys holds up even under different

Corresponding author: Angela D. Friederici (angelafr@cbs.mpg.de). training procedures, this paper must be considered highly relevant in the discussion of what constitutes the human language faculty. Indeed, Hauser, Chomsky and Fitch [4] have argued that the faculty to master recursiveness in sequences, which is a main feature of PSG, is the crucial parameter that distinguishes human from non-human primates. If the difference in processing these two grammar types is biologically based, the processing of these two grammars should be expected to be neuronally dissociable in the human brain. Either the processing of these two grammars is subserved by the same brain structure, but with different connection weights as a result of differential learning, or the processing of the two grammars is supported by separable brain structures.

From evolutionary neuroanatomy it is known that brain regions differ in their phylogenetic age. Such a phylogenetic differentiation holds not only for subcortical versus cortical structures, but has also been suggested for some adjacent cortical structures, for example the subcentral, the precentral and the inferior frontal gyrus [5]. Given this knowledge, we might predict that in humans the processing of these two grammars are subserved by separable brain structures that are adjacent but of different phylogenetic age, with the processing of the FSG being supported by the phylogentically older structure and the processing of the PSG by a phylogenetically younger structure.

So far, however, there is no brain imaging study in humans that has directly compared the processing of these two types of grammar. Within natural languages, which are always PSG, there are studies that can be classified as investigating either local phrase-structure violations or long-distance dependencies. As the aspect of local phrasestructure processing is the crucial parameter for FSG, and processing of hierarchical structure and long-distance dependencies is the determining aspect of PSG, an evaluation of the possible differences between local and

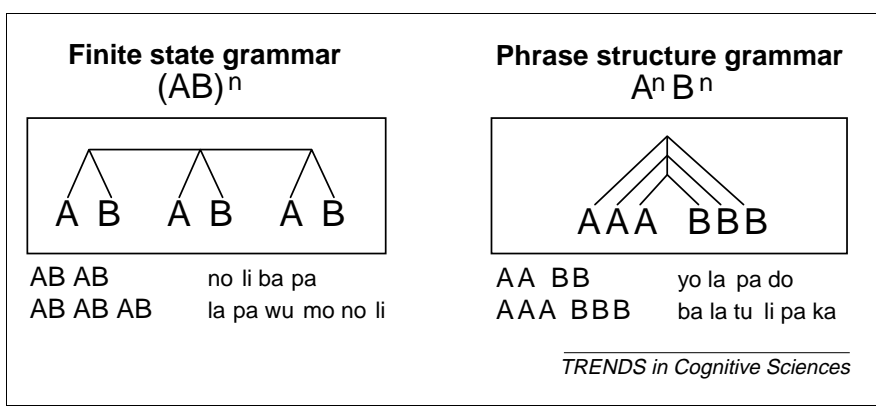

Figure 1. General structure and examples of stimuli in the Finite-State Grammar and the Phrase-Structure Grammar used by Fitch and Hauser [3]. 
non-local dependencies might provide a first indication about its neural underpinning.

\section{The role of Broca's area}

In the debate about the brain basis of syntax, Broca's area in the inferior frontal gyrus has been considered to play a major role [6], although exactly which aspects of syntactic processing are supported by this brain area remains an open question. Those brain imaging studies investigating long-distance dependencies involving syntactic movement and transformations have univocally reported the crown of Broca's area in the left hemisphere to be active [7-12]. By contrast, activation in left Broca's area has not been reported in studies evaluating different types of grammatical violations, such as word-order violation, agreement violation, verb-subcategorization violation [12-14], or local phrase-structure violation [15]. This last violation activated the left frontal operculum [15].

From these studies it appears that left Broca's area is involved when long-distance dependencies involving syntactic movement and transformational structures come into play, but not in the processing of local phrase structures (see Figure 2). The build-up of a local phrase structure during language perception seems instead to be subserved by the left posterior frontal operculum (subcentral gyrus), which according to Sanides [5] is most probably older evolutionary territory, derived from the insular pro-isocortex. This assumption, however, clearly needs further empirical support, as the only grammatical structure to have been investigated so far is a violated noun phrase in which an article is followed directly by a verb instead of a noun (for example: Das Eis wurde im gegessen/The ice-cream was in the eaten) [15]. In such a condition the transition probability between a determiner (the) and a verb (eaten) is zero and can, therefore, be detected by a very simple mechanism by which the incoming element is mapped onto the determiner-phrase template generated by the determiner. Other studies that have tested local word-order violations leading to a similarly low transition probability also report left opercular activity $[16,17]$. Fiebach et al. investigated a local word-order violation such as Vielleicht hat der Vater gegeben dem Sohn den Schnuller/Probably has the father given the baby the pacifier [17] (see Figure 2). As German is a verb-final language, the sentence becomes syntactically incorrect at the transition between the main verb and the next noun phrase. Given the sparse imaging data on the issue of local phrase-structure processing in natural languages this issue must remain open until further evidence is available.

\section{Evidence from artificial grammar learning}

There are, however, some imaging studies that have investigated rule-based language learning in natural and artificial languages. These studies consistently report activation in Broca's area for the learning of languagelike rules, whether a natural existing language or an artificial language mimicking natural grammars is used [18-20]. This brain region, however, was not activated when a rule that could not exist in any natural language was to be learnt [18]. Learning of finite-state artificial grammars instead activated Brodmann areas (BA) 38 and 47, but not the classical Broca's area (BA 44/45) [21-23]. BA 47 represents a presumably 'older' transition cortex that borders insular pro-isocortical areas.

Additional evidence for a differential neural network underlying the processing of FSG and PSG comes from electrophysiological data. Whereas violations in an FSG evoke a P300 [24], a component known to be a domaingeneral marker of deviance detection and processing of FSG violations elicits an early left anterior negativity for local phrase-structure violations and sustained frontal

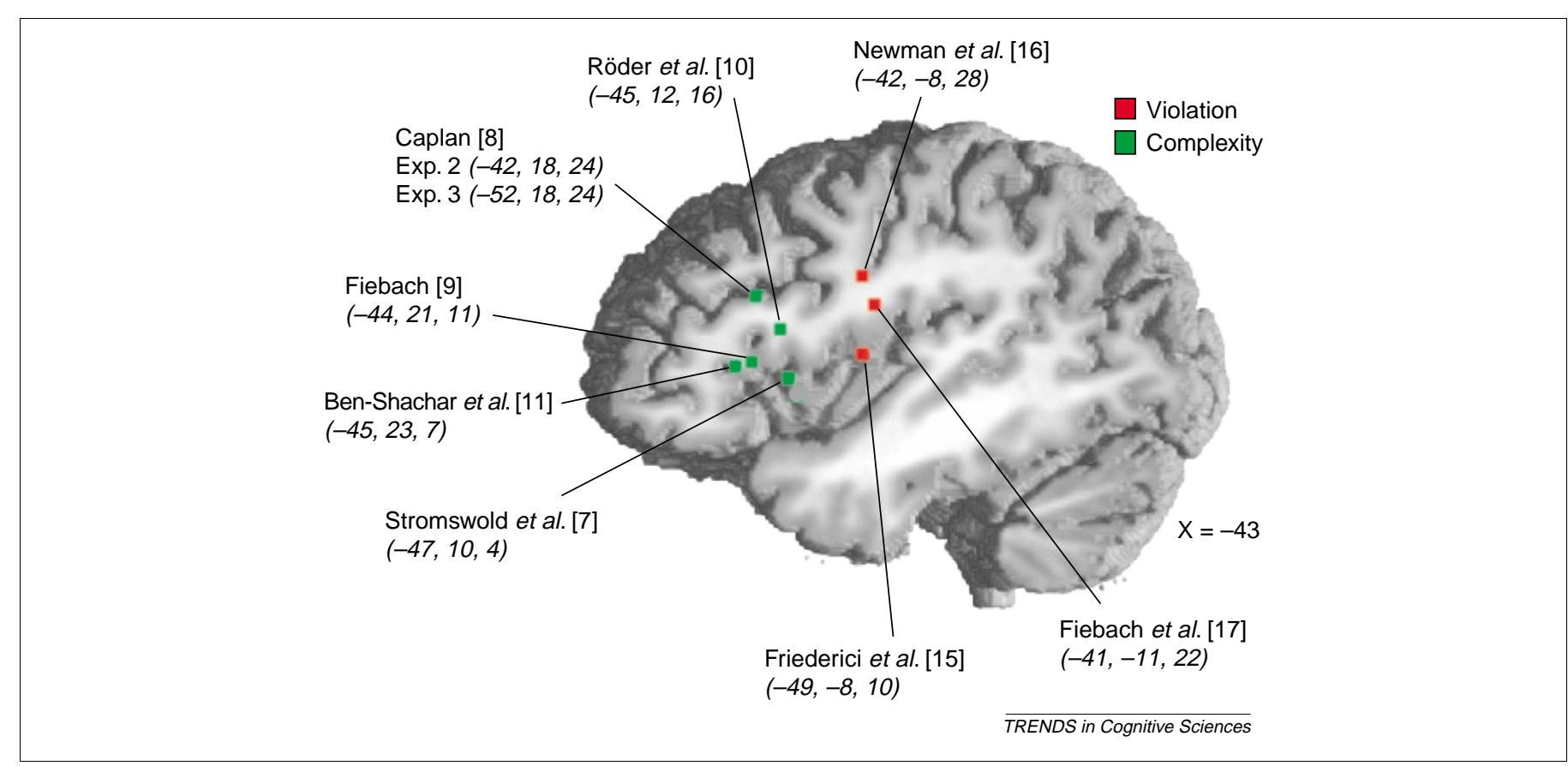

Figure 2. Activation of brain regions in different studies investigating syntactic processes according to the Talairach coordinates ( $x, y, z)$ given in the publications cited. Green: activation foci for studies investigating long-distance hierarchies and transformations; red: activation foci for studies evaluating local structural violations. 
negativity for long-distance dependencies [25]. Using a complex artificial grammar containing different types of phrase structure similar to natural languages, but (unlike natural languages) not including recursion, it was demonstrated that violations elicited an early anterior negativity followed by a P600 [26]. These data suggest that the brain response to violations is determined by the type of structure used, rather than the artificiality of the language.

\section{Conclusion}

In summary, the research reported by Fitch and Hauser [3] provides intriguing evidence for the notion that the ability to process PSG and thereby hierarchical structures is unique to humans. This claim receives support from studies in the neuroscience of language in humans, which reveal particular clusters of brain activation dependent on the type of structural information processed. Broca's area (BA 44/45) is most obviously activated during language processing when long-distance dependencies and transformational structures (i.e. hierarchical structures) are processed.

\section{References}

1 Lai, C.S.L. et al. (2001) A forkhead-domain gene is mutated in a severe speech and language disorder. Nature 413, 519-523

2 Enard, W. et al. (2002) Molecular evolution of FOXP2, a gene involved in speech and language. Nature $418,869-872$

3 Fitch, W.T. and Hauser, M.D. (2004) Computational constraints on syntactic processing in a nonhuman primate. Science 303, 377-380

4 Hauser, M.D. et al. (2002) The faculty of language: what is it, who has it, and how did it evolve? Science 298, 1569-1579

5 Sanides, F., ed. (1962) Die Architektonik des menschlichen Stirnhirns, Springer

6 Embick, D. et al. (2000) A syntactic specialization for Broca's area. Proc. Natl. Acad. Sci. U. S. A. 97, 6150-6154

7 Stromswold, K. et al. (1996) Localization of syntactic comprehension by positron emission tomography. Brain Lang. 52, 452-473

8 Caplan, D. (2001) Functional neuroimaging studies of syntactic processing. J. Psycholinguist. Res. 30, 297-320

9 Fiebach, C.J. (2001) Working Memory and Syntax During Sentence Processing, MPI Series of Cognitive Neuroscience, No. 23, Sächsisches Digitaldruck Zentrum, Dresden

10 Röder, B. et al. (2002) Brain activation modulated by the comprehension of normal and pseudo-word sentences of different processing demands: a functional magnetic resonance imaging study. Neuroimage 15, 1003-1014

11 Ben-Shachar, M. et al. (2003) The neural reality of syntactic transformations: evidence from fMRI. Psychol. Sci. 14, 433-440

12 Kuperberg, G.R. et al. (2000) Common and distinct neural substrates for pragmatic, semantic, and syntactic processing of spoken sentences: an fMRI study. J. Cogn. Neurosci. 12, 321-341

13 Meyer, M. et al. (2000) Neurocognition of auditory sentence comprehension: event-related fMRI reveals sensitivity to syntactic violations and task demands. Brain Res. Cogn. Brain Res. 9, 19-33

14 Moro, A. et al. (2001) Syntax and the brain: Disentangling grammar by selective anomalies. Neuroimage 13, 110-118

15 Friederici, A.D. et al. (2003) The role of left inferior frontal and superior temporal cortex in sentence comprehension: localizing syntactic and semantic processes. Cereb. Cortex 13, 170-177

16 Newman, S.D. et al. (2003) Differential effects of syntactic and semantic processing on the subregions of Broca's area. Brain Res. Cogn. Brain Res. 16, 297-307

17 Fiebach, C.J. et al. Distinct neural correlates of legal and illegal word order variations in German: how can fMRI inform cognitive models of sentence processing? In The On-Line Study of Sentence Comprehension (Carreiras, M. and Cifton, C., eds), Psychology Press (in press)

18 Musso, M. et al. (2003) Broca's area and the language instinct. Nat. Neurosci. 6, 774-781

19 Opitz, B. and Friederici, A.D. (2003) Interactions of the hippocampal system and the prefrontal cortex in learning language-like rules. Neuroimage 19, 1730-1737

20 Tettamanti, M. et al. (2002) Neural correlates for the acquisition of natural language syntax. Neuroimage 17, 700-709

21 Fletcher, P. et al. (1999) Learning-related neuronal responses in prefrontal cortex studied with functional neuroimaging. Cereb. Cortex 9, 168-178

22 Seger, C.A. et al. (2000) Neural activity differs between explicit and implicit learning of artificial grammar strings: an fMRI study. Psychobiology 28, 283-292

23 Strange, B.A. et al. (2001) Anterior prefontal cortex mediates rule learning in humans. Cereb. Cortex 11, 1040-1046

24 Baldwin, K.B. and Kutas, M. (1997) An ERP analysis of implicit structured sequence learning. Psychophysiology 34, 74-86

25 Friederici, A.D. (2002) Towards a neural basis of auditory sentence processing. Trends Cogn. Sci. 6, 78-84

26 Friederici, A.D. et al. (2002) Brain signatures of artificial language processing: evidence challenging the 'critical period' hypothesis. Proc. Natl. Acad. Sci. U. S. A. 99, 529-534

Book Reviews

\section{Is there only one way to become sapiens?}

How Homo became sapiens: on the evolution of thinking by Peter Gärdenfors. Oxford University Press 2003 . $£ 25.00$ (250 pp.) ISBN 0198528507

\section{Josep Call}

Max Planck Institute for Evolutionary Anthropology, Leipzig, Germany

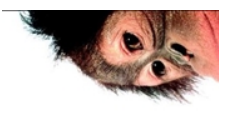

The evolution of cognition is an area that has received much attention in recent years. Gärdenfors has written a thoughtprovoking and easy-to-read book on the evolution of human cognition. The book is presented as a dialogue between an imaginary monkey called Egon that is eager to point out, often with a dose of sarcasm, potential loopholes in the reasoning of Gärdenfors as he revises a draft of his manuscript.

This book combines insights from animal behavior, archaeology, psychology, philosophy and neuroscience all in the quest for the understanding of the evolution of human thinking. I particularly enjoyed the discussion on the interplay of sensation, perception and imagination. Gärdenfors provides a vivid account of how cognition creates 'reality' out of the internal and external inputs. His discussion about the role of simulation and integration from various sensory inputs was extremely insightful. 